\title{
Éloge lyrique et digression : modèle rhétorique / pratique poétique
}

\section{Nathalie Dauvois}

\section{(2) OpenEdition}

1 Journals

Édition électronique

URL : http://journals.openedition.org/rhetorique/698

DOI : 10.4000/rhetorique.698

ISSN : 2270-6909

Éditeur

UGA Éditions/Université Grenoble Alpes

Édition imprimée

ISBN : 978-2-37747-062-4

\section{Référence électronique}

Nathalie Dauvois, «Éloge lyrique et digression : modèle rhétorique / pratique poétique ", Exercices de rhétorique [En ligne], 11 | 2018, mis en ligne le 18 octobre 2018, consulté le 12 septembre 2020. URL : http://journals.openedition.org/rhetorique/698 ; DOI : https://doi.org/10.4000/rhetorique.698

Ce document a été généré automatiquement le 12 septembre 2020.

\section{(c) (1) () (2)}

Les contenus de la revue Exercices de rhétorique sont mis à disposition selon les termes de la Licence Creative Commons Attribution - Pas d'Utilisation Commerciale - Partage dans les Mêmes Conditions 4.0 International. 


\title{
Éloge lyrique et digression : modèle rhétorique / pratique poétique
}

\author{
Nathalie Dauvois
}

«C'est le vrai but d'un poëte Liriq de celebrer jusques à l'extremité celui qu'il entreprend de

louer. Et s'il ne connoist en lui chose qui soit dinne de grande recommandation, il doit entrer dans sa race, \& là chercher quelqu'un de ses aieus, jadis braves, \& vaillans : ou l'honnorer par le titre de son païs, ou de quelque heureuse fortune survenue soit à lui, soit aus siens ou par autres vagabondes digressions, industrieusement brouillant ores ceci, ores cela, \& par l'un louant l'autre, tellement que tous deus se sentent d'une méme louange.» Ronsard «Au lecteur » (Odes 1550)

«Digressiones autem sunt lyricorum propriae... "

Pigna, Poetica Horatiana, 1561, 36

1 Si Gérard Milhe Poutingon, dans son beau livre sur la digression, convoque à plusieurs reprises le passage de l'avertissement au lecteur des odes cité ici en exergue, il ne s'attarde pas sur le lien qui y est défini entre éloge et digression, s'intéressant davantage à la dimension réflexive des digressions opérées par Ronsard dans ses odes ${ }^{2}$. De leur côté, Alex Gordon ou Daniel Ménager, en analysant les procédés de l'éloge chez Ronsard, s'intéressent moins à la digression qu'au mouvement et à la dynamique propres à l'éloge, en insistant en particulier sur le parcours des "lieux». Nous voudrions donc ici tenter d'approfondir cette relation entre éloge lyrique et digression, non seulement en mettant en lumière la corrélation entre le modèle rhétorique issu principalement de Quintilien (Institution oratoire IV, 3) et le modèle pindarique dont se 
réclame Ronsard, mais aussi en montrant le rôle que jouent en la matière les commentaires humanistes du début de l'épître aux Pisons autant que des odes épidictiques d'Horace. L'épître aux Pisons s'ouvre en effet par des considérations sur la composition du poème très souvent rattachées par les commentateurs humanistes à une poétique de la digression qui engage de manière plus générale le lien entre invention, disposition et élocution poétiques et permet de comprendre l'histoire et l'évolution de cette poétique, des premiers commentaires comme celui de Josse Bade, qui se situe à cet égard dans la droite ligne des arts poétiques médiévaux étudiés par Danielle James-Raoul ${ }^{3}$, à Minturno et Lambin.

\section{Rhétorique et poétique de la digression : modèle rhétorique/ modèle poétique}

2 Le livre IV de l'Institution oratoire de Quintilien porte sur l'ordre du discours, notamment judiciaire, sur sa dispositio. Or, il consacre un chapitre entier, entre le deuxième chapitre sur la narratio et le quatrième sur la confirmatio, à la digression, puisque, dit-il, c'est l'usage de certains rhéteurs, après la narration, de se lancer dans un excursus brillant à partir de lieux communs, pour susciter l'admiration (IV, 3, 2). Après avoir condamné cette pratique, et cette localisation spécifique, Quintilien ajoute qu'une digression est partout bien venue, à chaque moment du discours, à condition d'être pertinente, de servir la cause défendue ${ }^{4}$. Il va même jusqu'à donner à la digression une extension remarquable puisqu'il précise qu'il faut considérer comme telle tout ce qui ne relève pas des cinq parties à proprement parler du discours (exorde, narration, confirmatio, refutatio, péroraison). Sont donc définies comme digressions tout appel aux sentiments des juges, toute amplification, toute atténuation, tout ce qui donne de l'agrément au discours et l'orne ( $q$ quae maxime jucundam et ornatam faciunt orationem ${ }^{5} »$ ). C'est bien ce qui la rend particulièrement appropriée au genre démonstratif dont le but est de plaire et explique qu'on la retrouve dans le livre IX sur l'élocution, comme figure de pensée ${ }^{6}$. Les digressions introduisent elles-mêmes souvent dans le genre judiciaire des éléments de discours démonstratifs, ainsi de Cicéron insérant dans les Verrines un éloge de la Sicile ${ }^{7}$, quand elles ne développent pas les lieux communs de l'éloge.

3 Érasme reprend et développe ces éléments dans le second livre du De Copia consacré à la copia rerum, en en faisant un mode d'amplification à part entière ${ }^{8}$. La digression constitue en effet la sixième méthode d'amplification du sujet (après notamment la division et la description): "Superioribus affinis sexta locupletandi ratio quam Graeci $\pi \alpha \rho \varepsilon \kappa \beta \alpha ́ \sigma \iota \varsigma$, Latini partim egressionem, alii digressionem, nonulli excursus appellant ${ }^{9} "$, «Proche des procédés précédents, se trouve la sixième manière d'amplifier que les Grecs appellent parekbasis et les Latins, pour certains digressio pour d'autres egressio, pour d'autres excursus». Il y explique que la digression est surtout adéquate pour introduire des descriptions et développer les lieux communs de l'éloge des vertus et du blâme des vices.

Or ce sont ces développements que reprennent les premiers commentateurs humanistes à propos des v. 14-23 de l'ars poetica, en appliquant au poème les éléments d'analyse proposés par Quintilien pour les discours en prose et en distinguant à sa suite bonne et mauvaise digression. Les premiers commentateurs humanistes analysent en effet le célèbre début de l'épître aux Pisons sur la chimère poétique comme traitant de la disposition, de l'ordo, tout comme le livre IV de Quintilien, et lisent Horace à travers 
la grille d'analyse de ce dernier. À propos des v. 14-16 de l'épître aux Pisons où Horace se moque des poètes cherchant à rendre leur poème plus brillant en l'amplifiant par des ornements annexes et inappropriés :

Inceptis grauibus plerumque et magna professis

purpureus, late qui splendeat, unus et alter

adsuitur pannus (Horace, ars poetica, v. 14-16)

A un exorde issant de haute vois,

Et qui promet grans choses, maintes fois

Sont attachez mainz ornemens de lustre

Pour faire l'euvre apparoir plus illustre ${ }^{10}$.

Josse Bade reprend la condamnation par Quintilien de la recherche d'ostentation des rhéteurs et lit ce passage d'Horace comme une condamnation de la digressio « inepta »:

[14] Nunc reprehendit eos qui propter ostentationem ingenii sui [...] inutiliter digrediuntur aut qui grauem materiam nimis deliciosis egressibus in lasciuiam trahunt ${ }^{11}$.

Il reprend ensuite ceux qui pour faire montre de leur ingéniosité font des digressions inutiles ou qui traitent un sujet élevé de manière plaisante par des

digressions trop raffinées.

Il se situe ici dans la lignée d'un Jean de Garlande ou d'un Mathieu de Vendôme lisant ce début de l'ars poetica comme une série de préceptes concernant les différents vices de poésie ${ }^{12}$ dont celui d'incongrua digressio ${ }^{13}$. Mais la digression est ici condamnée non seulement pour cette recherche de brillant ostentatoire (et parce qu'elle s'écarte trop $\mathrm{du}$ sujet) mais parce qu'elle introduit une rupture stylistique. Si Bade développe, d'après Quintilien, les règles de la digressio apta qui consiste à ne pas s'écarter du sujet mais à être au service de la preuve, d'une transition, ou de l'éclat du style («probandi alicuius gratia, transitionis gratia, apparatus gratia $\left.{ }^{14} »\right)$; il en spécialise en effet l'application poétique par des règles d'aptum, de decorum, de pertinence ${ }^{15}$ plus spécifiquement stylistiques. Là est bien la spécification poétique qu'impose le texte même d'Horace (cf. ars poetica, v. 14: "inceptis gravibus...») aux premiers commentateurs ${ }^{16}$. De même, les exemples développés dans les vers suivants invitent à relier les deux méthodes d'amplification analysées successivement par Érasme, la digression et la description.

[...] cum lucus et ara Dianae

et properantis aquae per amoenos ambitus agros

aut flumen Rhenum aut pluuius describitur arcus;

sed nunc non erat his locus. (Horace, ars poetica, v. 16-19)

Quant on decrit la forest decoree,

Avec l'autel de Diane honnoree,

Ou le circuit du ruisseau ondoiant

Faisant son bruit par le pré verdoiant,

Ou bien le Rhin gracieux \& doux fleuve,

Ou l'arc du ciel qu'on voit avant qu'il pleuve:

Mais quoi cela ici n'est pertinent. (trad. Peletier, op. cit., v. 29-35)

Ce point est celui qu'avait retenu et développé Landino, le premier commentateur humaniste d'Horace, sensible dans tout ce début de l'ars au rapprochement entre poétique et peinture en même temps qu'à ce critère de la cohérence stylistique de la description-digression avec le reste du poème :

Intollerabile uitium est eorum poetarum, qui cum aliquid grauitate plenum scribendum susceperint, quod aut ad deos immortales aut heroas, aut ad praestantissimos uiros pertineat, ita in eo poemate uersari, ut quaedam minora in eo optime describas. In summa autem rei quod poetarum proprium est decorum non serues ${ }^{17}$.

Un défaut intolérable chez les poètes est de commencer à écrire sur un sujet d'importance, comme tout ce qui touche aux dieux immortels ou aux héros ou à des 
personnages exceptionnels, et de consacrer la suite du poème à décrire avec art des broutilles. En somme de ne pas préserver, ce qui est la qualité essentielle des poèmes, le decorum.

Parrasio en propose en quelque sorte une synthèse en opposant les digressions inopportunes («egressiones importunas») au savoir-faire de Virgile qui n'introduit jamais de digressions-descriptions de ce type, à la différence de Lucain :

Virgilius Georgicorum libro tert. Sic pestilentiam describit, ut nec discedat a materia, nec uagetur diutius. Idem facit in fama, \& in suis catalogis. Lucanus in prodigiis, massiliensi nemore, catalogis, magiaque nimius est. In hoc genere sunt omnes locorum, oppidorum, temporum descriptiones, quae si nimium luxuriarint, sunt damnandae ${ }^{18}$.

Virgile au troisième livre des Géorgiques décrit ainsi la peste, sans s'écarter de son sujet, ni s'attarder trop longuement. Il fait de même à propos de Fama et dans ses listes. Alors que Lucain, aussi bien dans sa description des prodiges que du bois sacré de Marseille, que dans ses listes ou à propos de magie, s'étend trop. Dans ce genre, toutes les descriptions de lieux, de places fortes, de tempêtes, si elles sont trop détaillées, sont à proscrire.

Il étend cette condamnation des digressions-descriptions qui visent à l'ostentation, de la disposition à l'élocution: "Nec minus digressio sit in eloquutione, \& quaedam poetica euagatio, cum plus dicimus quam conueniat ${ }^{19}$ ", "On peut parler aussi s'agissant de l'élocution de digression et en quelque sorte de divagation poétique quand nous en disons plus qu'il ne faut ».

6 Si ce début de l'ars permet ainsi aux commentateurs de spécifier dans une certaine mesure le cas de la digression poétique, il leur permet avant tout d'édicter des règles qui s'appliquent à la poésie en général et notamment à l'épopée, selon le modèle d'Homère, qu'Érasme loue comme maître incontesté de description ${ }^{20}$, et de Virgile. La plupart des exemples développés par les commentateurs sont en effet pris à l'Enéide, les digressions citées étant le plus souvent, dans la lignée des exemples donnés par Horace, des descriptions venant interrompre le fil de la narration. Et même si le précepte de pertinence stylistique tel que le reformule par exemple Landino pourrait sembler s'appliquer aussi bien aux odes et aux hymnes consacrés aux Dieux et aux héros, tous les exemples qu'il prend à la suite sont virgiliens.

7 Au fur et à mesure du siècle cependant, quand les commentaires plus aristotéliciens viennent recentrer ce début de l'ars sur la question de la fabula et des épisodes, assimilant digression et épisode ${ }^{21}$, un autre modèle poétique de la digression vient relayer celui-ci, les poèmes lyriques épidictiques d'Horace étant dorénavant eux aussi commentés à la lumière de leurs modèles grecs et notamment ici du modèle pindarique.

\section{Du modèle virgilien au modèle pindarique : digression, variété et genre épidictique}

Comme l'a montré Jean-Eudes Girot, le commentaire de Lonicer sur les épinicies pindariques ${ }^{22}$ joue un rôle essentiel dans la promotion et la compréhension du poète grec et en particulier du mode de composition de ses poèmes. Or Lonicer commente à plusieurs reprises les digressions de Pindare qui caractérisent selon lui sa manière. La première antistrophe de la troisième pythique qui introduit le mythe de Coronis (lequel constitue une bonne partie du développement de l'ode) est ainsi commentée, sobrement, en ces termes: " More suo poeta digreditur ${ }^{23}$ ", «Digression, selon l'habitude du poète ». De même, commentant la neuvième pythique - dont l'ode à la Reine de 
Ronsard (Odes 1550, I, $2^{24}$ ) par exemple, reprend l'essentiel du développement mythique sur Cyrène en le transposant à la Florence des Médicis -, Lonicer parle à propos du début du développement mythique au v. 5 d'excursus («excurrit ad Patriae Cyrenes originem $^{25}$ ", « excursus sur l'origine de Cyrène ») avant de marquer 70 vers plus loin la fin de ce développement par ces mots: "finita digressione de Cyrenes ortu a Nympha Cyrene Hypsei filia, etc., redit ad institutum, victoremque Telesicratem a victoria Pythia praedicat ${ }^{26} »$.

9 L'analyse de Lonicer correspond bien au développement de certaines odes de Ronsard où la narration mythique n'est pas considérée comme le cœur de l'ode ou de l'hymne mais comme une digression par rapport à l'éloge de la personne et de ses vertus, « riche matière » (Pythiques IX, v. 76²7). Cette richesse de la matière elle-même est associée non sans paradoxe apparent, selon le commentaire de Lonicer sur ce passage, à la brevitas, car le propre des lyriques est de savoir développer certains points à propos ${ }^{28}$. Cela permet de comprendre comment sont reliées poétique de la digression et poétique épidictique lyrique. Le poète lyrique a pour caractéristique de déborder le cadre de l' ordo, d'une disposition systématique des lieux de l'éloge, par l'élan d'une invention fondée sur la vagabonde digression comme amplification ponctuelle, choisie, appropriée.

La façon dont les odes d'Horace sont elles-mêmes analysées par les commentateurs et théoriciens de la seconde moitié du XVI ${ }^{\mathrm{e}}$ siècle comme de dignes imitations de cette poétique lyrique pindarique est éclairante à cet égard sur la façon même dont une poétique de la digression au service de l'éloge lyrique s'impose au tournant du siècle en France. Minturno, dans son De Poeta (1559), consacre tout un livre, le livre V, à la poésie lyrique qu'il appelle mélique, ce qui en fait un des théoriciens majeurs de la poésie lyrique à la Renaissance, comme l'a montré Gustavo Guerrero ${ }^{29}$. Horace et ses odes y constituent le modèle de référence. Or après avoir montré que la poésie lyrique participait elle aussi de la mimesis - les poèmes constituant autant d'actes de langage (de l'éloge au conseil à l'action de grâce ${ }^{30}$ ), de mimesis d'action -, Minturno s'arrête plus particulièrement sur l'art d'Horace dans l'éloge, sur la façon dont il est modelé sur celui de Pindare précisément par son art de la digression:

A materia quoque suscepta digreditur, quod genus est, cum Horatius a laudibus Augusti deflectit ad landandum Reguli virtutem, aut tum invocata Calliope ad dicendum, quae sibi obvenerant, ad belli narrationem, quod cum Diis Gigantes gesserunt, egreditur. Interiicit vero Pindarus plerumque quae sint praeter propositum genus ${ }^{31}$.

Il s'éloigne du sujet qu'il avait entrepris : par exemple, Horace passe de l'éloge d'Auguste à l'éloge du courage de Régulus [Carm. III, 5], ou bien, après avoir invoqué Calliope, il fait une digression sur sa propre biographie, ou raconte la bataille des Géants avec les Dieux [Carm. III, 4]. Pindare lui-même intercale souvent des développements qui débordent son propos initial.

Analyse remarquable qui fait de la digression, la marque même de l'éloge lyrique, son mode de développement de la matière. Nous trouvons des analyses comparables chez Denys Lambin, ami de Ronsard et son contemporain. Lambin, qui publie à son retour d'Italie, en 1561, l'édition commentée d'Horace qui lui vaudra la même année sa chaire d'éloquence latine au collège royal et l'année suivante son poste de lecteur royal de grec, se plaît en effet à souligner tout ce que doit Horace aux lyriques grecs et en particulier à Pindare, notamment dans ces procédés digressifs de l'ode. Ce qui lui permet de mettre en lumière la diversité des modes de la digression. Il ne rapproche pas seulement en effet la fin de l'ode III, 3 où Horace rappelle à l'ordre sa Muse qui vient de narrer un épisode de la guerre de Troie (v. 70 : «quo Musa tendis ? ", « où vas-tu 
ma Muse?») de deux passages semblables de Pindare dans les Néméennes et les Olympiques,

[...] Sic Musam reprimit Ode III. lib. 3. [v. 70-71] Quo Musa tendis ? desine peruicax Referre sermones Deorum. sic Pindarus in medio carmine seipsum, ab eo, quod instituerat, aberrantem, aut ad ea, quae ad rem susceptam minus pertinebant, digredientem reprimit, \&

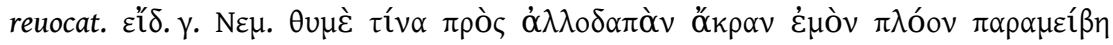

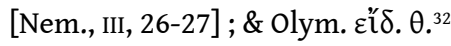

Il rappelle ainsi à l'ordre sa Muse dans l'ode 3 du $3^{e}$ livre, «Où vas-tu ma Muse ? cesse de t'obstiner à rapporter les discours des dieux »; de même Pindare au milieu de son poème se réprimande et rappelle lui-même à l'ordre alors qu'il est en train de dériver loin de son propos initial vers des considérations qui ont peu de rapport avec le sujet initial "Mais ô mon âme, vers quel promontoire étranger vas-tu égarer mon navire?". ${ }^{33}$ Et Olympique IX, etc.: ...

Type de développement et de rappel dont Ronsard comme Du Bellay se souviennent soulignant de même les vagabondages de leur Muse, ainsi du célèbre exemple de l'ode à Michel de L'Hospital :

$\mathrm{Ha}$, chere Muse, quel Zephyre

Souflant trop violentement

A faict ecarter mon navire, etc. (Ode à Michel de l'Hospital, V, 8, 693-695 $5^{34}$ ),

rappel qui sert de transition entre la narration mythique et le retour à l'éloge des vertus propres de L'Hospital. Mais le commentaire de Lambin illustre aussi la variété de ce type d'excursus, qui introduisent également dans le cours de l'ode épidictique des maximes, des sentences à valeur de vérité générale, à l'instar de Pindare là encore. Ainsi à propos de l'ode II, 13 :

Quid quisque uitet, \&c.] [13] Digreditur ad sententiam generalem \& uniuersam, neminem sibi in posterum ita cauere posse, ut non improuiso genere mortis extinguatur. quam \& Pindarus posuit Olymp. ${ }^{35}$ [il cite Olymp., II, 30-31 où Pindare introduit une sentence de vérité générale].

Il digresse vers une maxime générale et universelle : Personne ne peut se prémunir du futur au point d'être à l'abri d'une mort inopinée. De même Pindare dans la deuxième Olympique...

11 Ronsard là encore ne procède pas autrement en insérant des passages gnomiques dans ses odes épidictiques, passages gnomiques qui permettent assez systématiquement de passer de l'éloge des vertus particulières à l'éloge de la vertu en général. Nous retrouvons ainsi dans les passages digressifs de ses odes, l'ensemble des lieux qui caractérisent l'éloge, narration, éloge gnomique des vertus. Les digressions sur le modèle pindarique permettent ainsi d'amplifier et d'enrichir l'éloge par déplacement, dérivation, comme le formule clairement Ronsard dans son épitre au lecteur ;

C'est le vrai but d'un poëte Liriq de celebrer jusques à l'extremité celui qu'il entreprend de louer. Et s'il ne connoist en lui chose qui soit dinne de grande recommandation, il doit entrer dans sa race, \& là chercher quelqu'un de ses aieus, jadis braves, \& vaillans: ou l'honnorer par le titre de son païs, ou de quelque heureuse fortune survenue soit à lui, soit aus siens ou par autres vagabondes digressions, industrieusement brouillant ores ceci, ores cela, \& par l'un louant 1 'autre, tellement que tous deux se sentent d'une méme louange ${ }^{36}$.

12 Il ne s'agit pas seulement ici d'un parcours des lieux de l'éloge, mais bien de déplacements par contiguïté. L'ensemble des procédés de développement propres à l'éloge deviennent ainsi proprement lyriques par ce mouvement même de la digression, de la digression comme variation. Une bonne part du travail d'écriture des odes passe par cette amplification digressive, et par cet enchaînement de passages descriptifs, narratifs et gnomiques. Or ces narrations mythiques et ces maximes générales, définies 
comme autant de divagations-digressions, constituent la matière même d'une ode lyrique épidictique définie par cette dynamique d'amplification et de variation, mais aussi par cette aptitude à passer d'un éloge particulier à un éloge général, à ramener la circonstance à un modèle de vertu, de bravoure, d'éloquence, etc, ce qui est le principe même de l'écriture épidictique, qui vise à faire échapper le héros loué au temps de la circonstance pour le faire passer dans l'univers du mémorable garanti à la fois par les récits mythiques et par les sentences gnomiques ${ }^{37}$. Cette dynamique correspond ainsi à la vocation même de ce genre poétique et démonstratif qui est d'inciter à la vertu par l'exemple, autant que de plaire par la variété. Ronsard l'affirme un peu plus haut dans la même épître, mettant en relation variété ${ }^{38}$ et inconstance, admirable variété de la nature et du modèle de l'ode pindarique :

[...] je m'assure qu'ils ne me sçauroient accuser, sans condamner premierement Pindare auteur de telle copieuse diversité, \& oultre que c'est la sauce, à laquelle on doit gouster l'ode. Je suis de cette opinion que nulle Poësie se doit louer pour acomplie, si elle ne ressemble la nature, laquelle ne fut estimée belle des anciens, que pour estre inconstante, \& variable en ses perfections ${ }^{39}$.

Il redit un peu plus loin son admiration pour les «admirables inconstances» de Pindare. Ronsard prend bien soin d'ailleurs dans cette même épître, quelques lignes plus haut, d'opposer ce haut modèle de l'ode pindarique, digressif et varié, au modèle du sonnet amoureux, à sujet unique et fondé sur la continuité du propos :

Je ne fai point de doute que ma Poësie tant varie ne semble facheuse aus oreilles de nos rimeurs, \& principalement des courtizans, qui n'admirent qu'un petit sonnet petrarquizé, ou quelque mignardise d'amour qui continue tousjours en son $\operatorname{propos}^{40}$.

Ce lien entre modalités de développement de l'ode et digression se retrouve par exemple chez Le Tasse toujours à partir d'exemples d'Horace et de Pindare ${ }^{41}$. Or il détermine d'autant mieux le lien entre éloge et digression lyriques qu'il est fondé, comme là encore le commentaire de Lambin le met en lumière, sur une conception particulière de la poésie lyrique, comme poésie inspirée. Lambin fait en effet assez systématiquement le lien entre digression, éloge lyrique et fureur poétique.

\section{Digression, éloge et fureur poétique}

13 Lambin et Pigna publient tous deux leur commentaire ou paraphrase d'Horace à ce tournant des années 1560 qui voit la Poétique d'Aristote faire un retour en force, au détriment de la catégorie lyrique que le modèle italien avait contribué jusqu'alors à mettre en avant. Eux-mêmes poètes ou visant un public de poètes, ils s'attachent à spécifier ce lien entre digression et éloge lyrique en le fondant sur la théorie de l'inspiration poétique. À propos de la deuxième ode du troisième livre, Lambin montre ainsi qu'Horace s'éloigne de son premier sujet qui est un éloge de la vertu de tempérance pour introduire un éloge du silence, sans revenir ensuite à son premier sujet. Et il explique ce mouvement de divagation, cette inconstance, par le transport poétique de l'inspiration et là encore rapproche en cela Pindare et Horace :

Est \& fideli [25] laudata abstinentia, \& animo ab omni cupiditate libero, ac soluto, rerumque humanarum contemptore, digreditur, ac, poene dicam, aberrat ad laudem silentii : neque postea reuertitur ad sermonem institutum. Est enim poëtarum, tanquam spiritu diuino afflatorum, \& mentis agitatione concitatorum, non semper in proposito sermone haerere: sed ad alia nonnunquam aberrare, sententiaque longe a principio diuersa carmen concludere, cuius rei exempla sunt in Od.13., lib.2. \& Od. 7. lib.1. \& Od. 3. eiusdem. \& alia multa. apud Pindarum autem quam plurima. 
Après avoir fait l'éloge de l'abstinence, et d'un esprit libéré de toute cupidité qui méprise les biens de ce monde, il introduit une digression et, j'irais jusqu'à dire une divagation, pour passer à l'éloge du silence : et il ne revient pas à son premier propos. C'est en effet la marque des poètes, comme inspirés par un souffle divin et agités par un transport de leur esprit, de ne pas toujours s'en tenir au fil de leur discours : mais de s'évader parfois vers d'autres sujets et de clore leur chant sur un propos très éloigné du premier, on en rencontre maints exemples chez Horace [...] et en grand nombre chez Pindare ${ }^{42}$.

Rien d'étonnant donc à voir Ronsard introduire les plus amples digressions, au point d'en faire la matière essentielle de son ode, dans l'ode à Michel de l'Hospital dont la narration mythique digressive centrale porte précisément sur la théorie de l'inspiration.

On retrouve cette relation établie entre digression et éloge lyrique par la fureur poétique chez Pigna dont la Poetica horatiana paraît la même année 1561 que les commentaires de Lambin. Après avoir, comme ses prédécesseurs, introduit un développement sur la digression à propos des v. 14-17, distingué les digressions maladroites de celles qui sont à louer dans les différents genres littéraires (à savoir les descriptions qui se caractérisent par leur enargeia ${ }^{43}$ ), et mentionné les digressions comme épisodes, il y revient à propos des vers 83-85 qui énumèrent la diversité des sujets propres à la poésie lyrique apte à chanter aussi bien les Dieux et les héros (« Musa dedit fidibus diuos puerosque deorum...", "La Muse a confié à la lyre les dieux et les enfants de dieux... ») que la " vita voluptuosa ». Or il ouvre son commentaire de ces vers par une caractérisation par le mode. Alors que les types de poèmes énumérés précédemment par Horace (comme l'épopée, l'élégie ou l'iambe) étaient caractérisés par l'adéquation d'un mètre à un sujet (respectivement, les gestes héroïques, la plainte ou l'invective), les vers lyriques, qui ont de multiples sujets, se caractérisent d'abord, écrit-il, par un élan de l'inspiration qui s'exprime sur le mode digressif, marque propre de ce genre, de sujet et de forme variés, où l'inspiration pourvoit à faire correspondre « heureusement » prosodie et propos ${ }^{44}$ :

Et quemadmodum materiae singulae suis propriis numeris supra sunt assignatae, ita quid fidibus agatur, hic est expositum. Digressiones autem sunt lyricorum propriae : nam cum eorum carmina efferantur, rebus quas suscipiunt, feliciter respondent. Siquidem ipsarum quaedam solet esse exultatio quae est admodum laudabilis. Musa dedit fidibus ${ }^{45}$...

Et de la même façon qu'à chaque sujet a été plus haut assigné son mètre spécifique, il expose ici ce qu'il en est, s'agissant des vers lyriques.. Les digressions sont le propre des lyriques,. En effet lorsque l'inspiration emporte leurs chants, ceux-ci s'adaptent avec bonheur aux différents sujets qu'ils prennent en charge. Puisqu'une sorte de transport est en général ce qui est considéré comme le plus louable dans ce genre de poèmes.

Telle est bien l'esthétique qui prime dans les premières odes de Ronsard de 1550 mais qui est vouée à une certaine transformation au fur et à mesure que sa production lyrique évolue du modèle des odes à celui des Hymnes et de Pindare à Marulle et aux Alexandrins, via notamment Catulle.

Nous avons vu plus haut en effet à quel point les premiers vers de l'épître aux Pisons imposaient le lien entre digression et description et comment Pigna d'emblée reliait enargeia, description et digression. Agnès Rees a étudié de manière approfondie dans sa thèse la façon dont ce modèle épique de digression descriptive envahissait certains poèmes lyriques des poètes de la Brigade, de la «Prosphonématique » de Du Bellay à l'ode à Michel de l'Hospital ${ }^{46}$ jusqu'aux Hymnes de $1555-1556^{47}$. Nous voudrions montrer 
ici qu'il ne s'agit plus seulement, dans les Hymnes des Saisons de 1563 notamment, de rivaliser avec l'épopée et son enargeia descriptive ${ }^{48}$.

Le lien entre description, digression et éloge lyrique nous semble en effet développé de manière particulière par Ronsard dans ses hymnes les plus inspirés. C'est notamment le cas de ceux qu'il place sous le signe de l'inspiration bacchique, dont témoigne par excellence non seulement le Dythyrambe mais l'Hymne de l'Automne. Or, comme l'a montré Mary Morrison ${ }^{49}$, ce rapport entre vision et inspiration est fondé notamment, dès les Folastries, sur l'imitation de Catulle via Muret. Il prend au demeurant tout son sens et toute son ampleur, dans la composition même d'un texte comme l'Hymne de l'Automne, dans ce rapport subtil entre les odes bacchiques d'Horace ${ }^{50}$ et la marqueterie du poème de Catulle sur les Noces de Thétys et Pelée qui fait la part belle à la description de la tapisserie de l'enlèvement d'Ariane par Bacchus (Catulle 64, notamment 251-264). Car la force de la réécriture ronsardienne y passe par un lien, renouvelé par l'inspiration bacchique elle-même, entre digression, description et éloge. Si le Dithyrambe est scandé par une série anaphorique de « je voy » et de «j'oy " ${ }^{51}$, l'Hymne de l'Automne, introduit par un long prélude métatextuel consacré à l'inspiration (« Tu seras du vulgaire appelé frenetique, / Insencé, Furieux, farouche, fantastique... $»^{52}$ ) est remarquable par l'abondance des digressions descriptives, des tableaux successifs qui le constitue, de la belle description de sa nourrice filant (v. 105-114) aux descriptions successives de l'antre de l'Auton (v. 171-208), de l'Aurore, du Ciel étoilé, puis du palais du Printemps (267-282), des filets de Zéphyre (289-299), du palais de l'Esté (303-326), puis de Nature (327-356) et enfin du char et du cortège de Bacchus (369-392). De même que les grandes odes pindariques font alterner digressions narratives et gnomiques, l' Hymne de l'Automne déploie un éloge en forme de parcours de lieux descriptifs qui culmine avec le tableau inspiré de Bacchus et son cortège.

Bien plus que de l'épopée, la démarche ronsardienne nous semble donc relever, des premières odes aux hymnes plus tardifs, d'une poétique de l'éloge lyrique comme parcours digressif. Si en 1563 les moyens et les modèles ne sont plus seulement horatiens, ils sont encore en quelque sorte programmés par Horace, du début de l'ars poetica à sa propre pratique dans les odes. La digression fournirait ainsi à Ronsard, au long de sa carrière, le mode opératoire privilégié pour traduire le mouvement même de l'inspiration.

\section{NOTES}

1. Nous citons l'édition de Paul Laumonier et alii, Ronsard, Euvres complètes, Paris, STFM, 1914-1975, 20 vol., dorénavant Lm, ici t. I, p. 48.

2. Voir G. Milhe Poutingon, Poétique du digressif. La digression dans la littérature de la Renaissance, Paris, Classiques Garnier, 2012, p. 209-217 où il analyse les « vagabondes digressions » de l'ode à Du Bellay et de l'Ode de la Paix jusqu'à l'ode à Madame Marguerite; cf. aussi p. 352 sur ce point.

3. D. James-Raoul, «La digression dans les Arts Poétiques du $12^{\mathrm{e}}$ et du $13^{\mathrm{e}} \mathrm{s}$. : aperçu théorique » dans La Digression dans la littérature et l'art du Moyen Âge, dir. C. Connochie-Bourgne, Senefiance $\mathrm{n}^{\circ} 51,2005$, p. 229-240. 


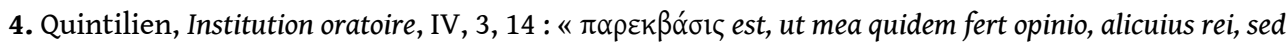
ad utilitatem causae pertinentis, extra ordinem excurrens tractatio. » « La parekbasis est, selon moi du moins, le traitement sous forme de digression, hors du plan naturel, d'un point quelconque, mais d'un point utile à la cause ». Nous citons le texte et la traduction de J. Cousin à la CUF, Paris, Les Belles lettres, 7 vol., 1975-1980. Voir H. Lausberg, Handbook of Literary Rhetoric : A Foundation for Literary Study, Leiden, Brill, 1998, § 340-342 pour une étude comparative avec les autres rhéteurs antiques sur ce point.

5. Quintilien, Institution oratoire, IV , 3, 15.

6. Voir Quintilien, Institution oratoire, IX, 1, 28 où Quintilien cite à ce propos le De Oratore de Cicéron, mais aussi IX, 2, 55 où il se demande s'il faut la compter parmi les figures, pour mieux confirmer, avec Cicéron, en IX, 2, 56, que brèves, les digressions en relèvent.

7. Ibid., IV, 3, 13.

8. Nous renvoyons ici aux analyses de J. Chomarat dans Grammaire et Rhétorique chez Érasme, Paris, Les Belles Lettres, 1980, t. 2, p. 740-741.

9. Érasme, De Duplici Copia verborum ac rerum, dans Opera omnia Desiderii Erasmi Roterodami, II, LB 82, éd. B. I. Knott, t. 6, Amsterdam, North Holland, 1988, p. 215. Nous traduisons, comme partout ailleurs, sauf mention contraire.

10. Nous citons la traduction de Jacques Peletier du Mans, L'Art poétique d'Horace traduit en vers français, éd. J. Vignes, dans Euvres complètes, Paris, Champion, 2011, I, p. 109, v. 25-28. Cf. la façon dont Sebillet file la métaphore horatienne du pannus, du morceau d'étoffe dans le chapitre 3 de son Art poétique qui développe abondamment la théorie horatienne du decorum (Traités de poétique et de rhétorique de la Renaissance, éd. F. Goyet, Paris, Librairie générale française, 1990, p. 59) : « Il regardera aussi soigneusement à joindre les unes choses aux autres proprement au progrès de son poème : et à y mettre les fins et commencements tant bienséants, qu'il ne soit repris comme le sot couturier faisant le capuchon de la cape du plus laid et mal uni endroit de la frise, et remplissant les quartiers de la robe noire, d'une pièce ou rouge ou verte. »

11. Nous citons le commentaire de Bade d'après l'édition des Opera d'Horace publiés à Paris par D. Roce et J. Petit pour Josse Bade en 1503, ici vol. 3, De arte poetica libellus, $3 \mathrm{v}^{\circ}$. Le lecteur en trouvera la transcription sur le site Renaissances d'Horace.

12. Voir sur ce point J.-Y. Tilliette, Des Mots à la Parole, Genève, Droz, 2000, p. 42 et sq., et le commentaire Materia dans l'édition de K. Friis-Jensen, «The Ars poetica in Twelfth-Century France ", Cahiers de l'institut du Moyen-Âge grec et latin, 60, 1990, p. 335 et sq. Voir pour l'ensemble des travaux du savant danois sur le sujet, The Medieval Horace, éd. K. M. Fredborg, M. SkafteJensen, M. Pade et J. Ramminger, Rome, Edizioni Quasar, 2015. Nous nous permettons enfin de renvoyer pour une analyse plus approfondie à notre article paru dans Camenae $n^{\circ} 13$ - octobre 2012 : «Decor, convenance, bienséance et grâce dans les arts poétiques français », ici p. 5.

13. Voir D. James-Raoul, op. cit., notamment note 43.

14. Commentaire de Bade, op. cit., $3 \mathrm{r}^{\circ}$.

15. Sur le caractère central de la notion de pertinence dans l'étude de la digression, voir G. Milhe Poutingon, op. cit., passim.

16. Cf. Aulo Giani Parrasio, A. Iani Parrhasii Cosentini in Q. Horatii Flacci Artem Poeticam Commentaria luculentissima, Naples, s.n. [Johann Sultzbach], 1531, fol. 10v : «[Inceptis grauibus] Magnum poematis uitium est ubi grandis proposita sit materia excessus facere, \& egressiones importunas, per locos praesertim communes. » ( "C'est un défaut grave dans un poème, après avoir introduit un sujet élevé, de partir dans des excursus et des digressions intempestives, qui prennent surtout la forme de lieux communs ».) On trouvera également la transcription de son commentaire sur le site Renaissances d'Horace.

17. Nous citons le commentaire de Landino, publié pour la première fois en 1482, dans l'édition par Fabricius des Opera d'Horace, Bâle, s.n. [H. Petrus], 1555, p. 917, également en transcription sur le site Renaissances d'Horace, ici p. 916. 
18. Parrasio, op. cit., $10 \mathrm{v}^{\circ}$.

19. Ibid.

20. Voir Érasme, De Copia, II, 164, à propos de la cinquième méthode d'amplification, la description, l'éloge d'Homère : "Hac virtute praecellunt cum omnes poetae, tum praecipue Homerus ", "C'est en quoi excellent tous les poètes, mais surtout Homère ». Voir J. Chomarat, Grammaire et Rhétorique chez Érasme, op. cit., p. 739.

21. Pour l'évolution des commentaires sur cette ouverture de l'ars poetica, de Bade aux commentateurs aristotéliciens, nous renvoyons à 0 . Millet, «Poétique, rhétorique et allégorie : les interprétations humanistes de la chimère horatienne (Art poétique, vers 1-13) ", Camenae 13 (octobre 2012).

22. Lonicer publie une première traduction de Pindare en 1528, et une traduction accompagnée d'un commentaire en 1535 : Pindari Poetae vetustissimi lyricorum facile principis, Olympia, Pythia, Nemea, Isthmia per Iona. Lonicerum latinitate donata : adhibitis ennarationibus... Bâle, Cratander, 1535. C'est l'édition que nous avons consultée.

23. Ibid., p. 191 ; cité par J.-E. Girot, Pindare avant Ronsard, Genève, Droz, 2002, p. 159.

24. Lm, I, p. 65-71.

25. Lonicer, op.cit., p. 175.

26. Ibid. p. 281 : « une fois achevée la digression sur la naissance de Cyrène, il revient de la Nymphe Cyrène fille d'Hypseus à son propos, et chante Télésicrate victorieux, pour sa victoire aux jeux pythiques. » Voir les analyses de J.-E. Girot, op. cit., p. 160.

27. "Les grandes vertus sont toujours une riche matière ", Pythiques, éd. et trad. A. Puech, Paris, Les Belles Lettres, 1966.

28. Lonicer, op. cit., p. 281 : «Brevitas enim Lyricis poetis propria est. [...]. Insigni vero loco, de occasione, haec amplificat. » (« La brièveté en effet est propre aux poètes lyriques. [...] Mais dans ce passage remarquable, s'agissant de l'à-propos, il amplifie ces points.») Il commente les vers suivants de Pindare, $9^{\mathrm{e}}$ pythique, éd. et trad. citée v. 77-78: « d'un vaste sujet faire briller quelques parties, voilà qui plaira aux oreilles savantes; en toutes choses, il faut l'à-propos avant tout ».

29. G. Guerrero, Poétique et poésie lyrique, Essai sur la formation d'un genre, Paris, Le Seuil, 2000.

30. Antonio Sebastiano Minturno, De Poeta, Venise, Rampazetto, 1559, p. 386-387.

31. Ibid. p. 388.

32. D. Lambin, Q. Horatius Flaccus ex fide atque auctoritate decem librorum manuscriptorum, opera Dionys. Lambini Monstroliensis emendatus: ab eodemque commentariis copiosissimis illustratus, nunc primum in lucem editus, Lugduni : Apud Ioann. Tornæsium 1561, p. 150.

33. Pindare, Néméennes, III, 26-27, trad. A. Puech, Paris, Les Belles Lettres, 1967.

34. Lm III, p. 157. Cf. par exemple Du Bellay, Vers lyriques xi, v. 57 : « Où vas-tu, Muse ?... » éd. M.D. Legrand, dans Euvres complètes, t. 2, sous la dir. d'O. Millet, Paris, Champion, 2003, p. 77.

35. Lambin, op. cit., p. 188-189. G. Milhe Poutingon (op. cit., p. 215) intègre cette insertion de gnômê dans sa liste de procédés digressifs.

36. Lm I, p. 48.

37. Nous nous permettons de renvoyer sur ce point à nos analyses dans N. Dauvois, Mnémosyne, Ronsard une poétique de la mémoire, Paris, Champion, 1992, notamment p. 23-28 et 93-114.

38. Sur la variété comme marque de poésie, voir Landino (op.cit., p. 917) à propos du v. 29 de l'ars poetica: "Qui uariare cupit] Virtus maxima est, poëma multa uarietate distinguere. Animum enim auditoris ex uarietate delectamus, \& attentum reddimus, \& ab omni fastidio remouemus. " ("La qualité par excellence est de savoir diversifier un poème par une grande variété. Par la variété nous charmons l'auditoire, nous le rendons attentif et lui évitons l'ennui. »)

39. Lm I, p. 47. Cf. Érasme, De Copia, I, ch. 8 (cité et traduit par J. Chomarat, Grammaire et Rhétorique chez Érasme, op.cit., p. 719) : "en toutes choses la variété a une telle force qu'absolument rien n'est assez brillant pour ne pas paraître terne sans le soutien de celle-ci. La nature elle aussi peut 
prendre plaisir par dessus tout à la variété, puisque dans l'immense foule des êtres (rerum) elle n'a rien laissé nulle part qu'elle n'ait dessiné avec un art admirable de la variété. »

40. Lm I, p. 47.

41. Voir lettre à V. Laurio, datée du 6 Septembre 1553 (Lettere, éd. A. Chemello, Bologne, Forni, 2002, vol. 2, p. 112-113), où il souligne que « il lirico cominciata la materia principale che s'ha proposto di trattare, e uscendo poi con la digressione, a le volte ritorna ne la materia principiata, a le volte finisce il suo poema nella digressione; il che si vede in Pindaro, et in Orazio in moltissimi lochi.» ("Le poète lyrique commence à traiter le sujet principal qu'il s'est proposé, puis s'échappe par une digression, et tantôt revient à son sujet premier, tantôt finit sur une digression. Comme on peut le voir chez Pindare et chez Horace en de très nombreux passages. ") Voir à ce propos l'article de G. Comiati : "Horace chez Bernardo Tasso : réécrire les odes en vers italiens ", dans Chacun son Horace, sous la direction de M. Jourde, J.-C. Monferran et N. Dauvois, Paris, Champion, 2018, p. 69-89.

42. Lambin, op.cit., p. 227. Cf. Ronsard, Responce aux injures, v. 872, sur la différence entre le poète et l'orateur qui toujours « suit le fil d'une chose », Lm XI, p. 160.

43. Giovanni Battista Pigna, Poetica horatiana, Venise, V. Valgrisi, 1561, p. 5 où il développe comme ses prédécesseurs l'impératif de decorum stylistique puis cite Aphtonius pour définir l' enargeia, toute la suite opposant des exemples de digressions-descriptions opportunes à des digressions « vitiosae ».

44. Cf. Montaigne, Essais, III, 9 (éd. Villey-Saulnier, Paris, PUF, 1988, p. 995) sur l'allure poétique "à sauts et à gambades » et ce lien entre inspiration et digression : "Le poete, dict Platon, assis sur le trepied des Muses, verse de furie tout ce qui luy vient en la bouche, comme la gargouille d'une fontaine, sans le ruminer et poiser, et luy eschappe des choses de diverse couleur, de contraire substance et d'un cours rompu ».

45. Pigna, op. cit., p. 36.

46. Voir A. Rees, thèse dactylographiée (à paraître), La Poétique de la vive représentation et ses origines italiennes, Reims, 2011 (en ligne sur le site de l'université de Reims), p. 348-351 sur l' enargeia des descriptions fluviales dans la " Prosphonématique »; et p. 417-418 sur l'ode à Michel de L'Hospital.

47. Cf. A. Rees, ibid., p. 306-309 sur l'ecphrasis dans les Hymnes; et son article "L'enargeia chez Ronsard : une poétique de la Fantasie (1555-1560) », dans Camenae 8, décembre 2010. Voir aussi Ph. Ford, Ronsard's Hymns. A Literary and Iconographical Study, Tempe (Arizona), Medieval and Renaissance Studies, 1997 ; et les livres fondateurs de P. Galand, Le Reflet des Fleurs. Description et métalangage poétique d'Homère à la Renaissance, Genève, Droz, 1994 et Les Yeux de l'éloquence. Poétiques humanistes de l'évidence, Caen, Paradigme, 1995.

48. Voir notamment sur ce modèle virgilien de la digression descriptive telle que le définissent Vida et à sa suite Peletier du Mans, A. Rees, "Poétiques de la "vive représentation" de Marco Girolamo Vida (1527) à Jacques Peletier du Mans (1555) », Italiques xii, 2009, p. 93-122.

49. M. Morrison, «Ronsard and Catullus », Bibliothèque d'Humanisme et Renaissance, 1956, XVIII, p. 240-274, notamment p. 265-267.

50. Voir Carmina, II, 19 et III, 25, odes marquées par ce mouvement de l'inspiration («Quo me rapis Bacche... », III, 25, 1) traduit dans une série de visions (« Bacchum... vidi », II, 19, 1-2).

51. Lm V, p. 53 et suiv.; voir l'incipit pour ce lien entre fureur et vision : «Tout ravy d'esprit je forcene / Une nouvelle fureur me mene, / D'un saut de course dans les bois, / Iach, ïach, j'oy la vois / Des plus vineuses Thyades / Je voy les folles Menades ». Cf. v. 11, 17, 27, 69, 79-80, etc., développant autant de digressions descriptives pour introduire l'éloge du dieu lui-même.

52. Lm XII, p. 49, v. 61-62. 


\section{AUTEUR}

NATHALIE DAUVOIS

Université Sorbonne Nouvelle - Paris 3 